\title{
Hybrid Super Resolution using SWT and CT
}

\author{
Deepa K Davis \\ Dept of Electronics and Communication \\ Federal Institute of Science and Technology \\ Ernakulam, India
}

\author{
Rajesh Cherian Roy \\ Associate Professor \\ Dept of Electronics and Communication \\ Federal Institute of Science and Technology
}

\begin{abstract}
Super resolution is the process of reconstructing high resolution(HR) image from multiple low resolution(LR) images of a scene. LR images are at a specific pixel shift from each other. High Resolution(HR) grids are formed from these LR images using Stationary Wavelet Transform (SWT) by image registration. Interpolation of the missing pixel in the HR grid is done using Curvelet Transform(CT). Experimental results show that our method has got high PSNR compared to Curvelet Transform based super resolution. Also, it is verified that there is no need of filter to avoid interpolation errors
\end{abstract}

\section{General Terms}

Stationary Wavelet Transform, Curvelet transform Keywords

Pixel Shift , Image registration, Interpolation

\section{INTRODUCTION}

In many, military and civilian applications, High Resolution (HR) images are desirable and always required. High Resolution refers to an image with a high level of information. Resolution is determined by the pixel density. In recent decades, charge-coupled device (CCD) and CMOS image sensors have been widely used in imaging systems. So a technique known as Super Resolution(SR) is used to enhance the resolution of an imaging system.

The basic principle of the SR technique is the increase in the resolution with the available multiple low resolution images. If the images are sub-pixel shifted, then each low resolution image contains independent information. So the information content is more if the SR image is formed from multiple low resolution images[1].

Super resolution reconstruction from multiframes is a relatively new problem.Tsai and Huang[2] were the first to super resolve a single $\mathrm{HR}$ frame from several $\mathrm{LR}$ frames(without blur).Saucer and Allebach[3] modeled super resolution as an interpolation problem with nonuniformly sampled data. They used projection onto convex set(POCS) algorithm to reconstruct the unknown values. Aizawa[4] also modeled super resolution as an interpolation problem with nonuniform sampling and used a formula related to Shannon's sampling theorem to estimate values on HR grid.Irani and Peleg[5] incorporated sensor blur into a set of linear equations, which is solved by iterative back projection method. Abdou [6] apllied a method to iteratively refine the $\mathrm{hr}$ estimate. Wavelet based interpolation for Super Resolution suggested by Nguyun and Milanfar[7] takes advantage of regularity in data to be interpolated. D L Ward [8] proposed an algorithm takes advantage of the redundancy of discrete wavelet transform to interpolate the registered half pixel shifted image. Anil and Jyoti [9] replaced the wavelet transform based interpolation with the Curvelet transform. Curvelet transform [10] is a multiscale pyramid with many directions and positions at each length scale. So compared to wavelet transform which has only three directions, curvelet transform is more advantageous to represent curves in images. This paper combines the advantages of both the Stationary wavelet transform and the curvelet transform. Here Stationary wavelet transform is applied to the low resolution frames and the image registration is carried out in respective sub bands. After this, interpolation is done in the Fast Discrete Curvelet Transform(FDCT) domain. FDCT is a multiscale geometric transform, which is a multiscale pyramid with many directions and positions at each length scale. The directional decomposition with anisotropic scale enables FDCT to capture the smoothness along the curves. Performance of the proposed algorithm shows improvement in PSNR

The organization of this paper is as follows; section 2 explains the proposed algorithm, section 3 shows the performance of the proposed algorithm and its comparison with the Curvelet transform based super resolution, section 4 gives conclusion

\section{PROPOSED ALGORITHM}

The proposed super resolution algorithm uses SWT sub bands for image registration and a curvelet transform based interpolation to form a single high resolution image. The two low resolution images are formed from the original image by half pixel shift of the original image in the vertical and horizontal directions. Then Stationary wavelet transform is applied on these low resolution images to form 8 sub bands. The four frequency components of each low resolution images are mapped on the high resolution grid respectively. Each high resolution grid is rotated 45 degrees to get better pixel correlation. Rotated grid is up sampled to generate space for missing pixel. Curvelet transform is applied to up sampled grid and interpolation is performed at the finest scale .Inverse Curvelet transform is applied to this interpolated curvelet coefficients. The interpolated grid is re-rotated back to its original orientation. Then inverse SWT is applied to these matrices to form the super resolved image

\subsection{Low resolution images using half pixel shift}

Traditional pixel-shifting technology enhances resolution by moving the image sensor typically half of a pixel between each image capture, sampling the object at more locations, and using these extra captured pixels directly as image data. Half pixel shift in two frames simulates the diagonal motion of a camera over an area. Fig .1 shows the pixel shift 

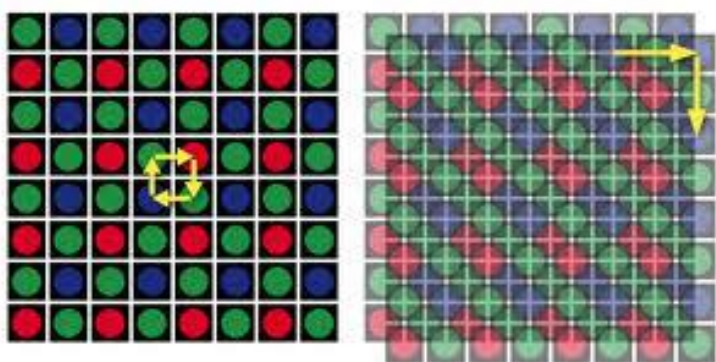

Figure 1 Half pixel shift

Two low resolution images are created from the original image by a half pixel shift in the $\mathrm{x}$ and $\mathrm{y}$ direction relative to one another. This fashions a Quincunx sampling pattern. Fig. 2 illustrates the pattern

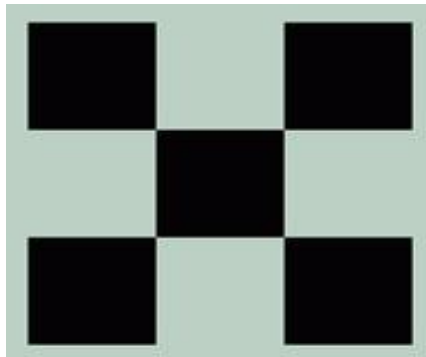

Figure 2 Quincunx pattern.

For an original image of size $256 \times 256$, two low resolution images $L R_{1}$ and $L R_{2}$ of size $128 \times 128$ are formed. Low resolution images take pixels from original image with purely even and odd valued indices

\subsection{Stationary Wavelet Transform}

Classical DWT suffers a problem that it is not translation invariant transform. Translations of the original signal lead to different wavelet coefficients. In order to overcome this and to get more complete characteristic of the analyzed signal the undecimated wavelet transform(stationary wavelet transform,SWT)[11] was proposed. The general idea behind it is that it doesn't decimate the signal. Thus it produces more precise information for the frequency localization. From the computational point of view the undecimated wavelet transform has larger storage space requirements and involves more computations. The SWT is an inherently redundant scheme as the output of each level of SWT contains the same number of samples as the input - so for a decomposition of $\mathrm{N}$ levels there is a redundancy of $\mathrm{N}$ in the wavelet coefficients.

Stationary Wavelet Transform is applied to each low resolution image. So for each low resolution image we have four sub bands. Therefore a total of 8 sub bands for these two low resolution images, $L R_{1}$ and $L R_{2}$. For $L R_{1}$ we have four sub bands: approximation $c A_{1}$, horizontal $c H_{1}$, vertical $c V_{1}$ and diagonal $c D_{1}$. For $L R_{2}$ we have $c A_{2}, c H_{2}, c V_{2}$ and $c D_{2}$

\subsection{Image Registration}

Each sub bands need to be mapped onto a common reference plane. This process is registration. Image registration deals with mapping corresponding points in each sub bands to the actual points in the sub bands of the original image and transforming data into one co-ordinate system.

Approximation sub band of each low resolution images $\mathrm{CA}_{1}$ and $\mathrm{cA}_{2}$ are combined on to a high resolution quincunx grid [11]. First pixel position of high resolution grid is filled with the first pixel in $\mathrm{CA}_{1}$, the second location will be the missing pixel location and the third location is filled with first pixel of $\mathrm{cA}_{2}$. Thus we got a high resolution grid of approximation sub band. Each approximation component in high resolution grid corresponds to an approximation component of high resolution image. High resolution grid of approximation component is designated as $\mathrm{Ha}\left(\mathrm{x}_{\mathrm{H}}, \mathrm{y}_{\mathrm{H}}\right)$ and the approximation components of two low resolution images as $\mathrm{Fa}_{1}\left(\mathrm{x}_{1}, \mathrm{y}_{1}\right)$ and $\mathrm{Fa}_{2}\left(\mathrm{x}_{2}, \mathrm{y}_{2}\right) \cdot \operatorname{Tr}($.$) is a transform for combining$ the approximation component of two low resolution images to form quincunx pattern, then

$$
\mathrm{Ha}\left(\mathrm{x}_{\mathrm{H}}, \mathrm{y}_{\mathrm{H}}\right)=\operatorname{Tr}\left(\mathrm{Fa}_{1}\left(\mathrm{x}_{1}, \mathrm{y}_{1}\right), \mathrm{Fa}_{2}\left(\mathrm{x}_{2}, \mathrm{y}_{2}\right)\right)
$$

where $x_{1}=2 x_{H}-1, \quad y_{1}=2 y_{H}-1$

$$
\mathrm{x}_{2}=2 \mathrm{x}_{\mathrm{H}}, \mathrm{y}_{2}=2 \mathrm{y}_{\mathrm{H}}
$$

The resultant image is a quincunx pattern of the approximation components of the desired high resolution image. Similarly the other sub bands are combined to form the other sub bands of desired high resolution grid.

\subsection{Image rotation}

Rotation performs a geometric transformation which maps the position $\left(\mathrm{x}_{1}, \mathrm{y}_{1}\right)$ of an input image onto a position $\left(\mathrm{x}_{2}, \mathrm{y}_{2}\right)$ in an output image by rotating it through a specified angle about an origin.

Here a $45^{\circ}$ rotation is applied to each sub bands changing its index from $\left(\mathrm{x}_{\mathrm{H}}, \mathrm{y}_{\mathrm{H}}\right)$ to $\left(\mathrm{x}_{\mathrm{rot}}, \mathrm{y}_{\text {rot }}\right)$. Each pixel in high resolution grid have to be transferred to rotated high resolution grid. This increases the pixel correlation between respective sub bands of $\mathrm{LR}_{1}$ andLR 2 . Fig. 3 demonstrates the rotation

Let $\mathrm{R}_{\text {rot }}$ denotes the $45^{0}$ rotation performed on high resolution grid of each sub band. Consider the high resolution grid of approximation component $\mathrm{Ha}\left(\mathrm{x}_{\mathrm{H}}, \mathrm{y}_{\mathrm{H}}\right)$. When we apply $\mathrm{R}_{\text {rot }}$ on $\mathrm{Ha}\left(\mathrm{x}_{\mathrm{H}}, \mathrm{y}_{\mathrm{H}}\right)$ we have

$$
\mathrm{H}_{\text {rot }}\left(\mathrm{x}_{\text {rot }}, \mathrm{y}_{\text {rot }}\right)=\mathrm{R}_{\text {rot }}\left(\mathrm{Ha}\left(\mathrm{x}_{\mathrm{H}}, \mathrm{y}_{\mathrm{H}}\right)\right)
$$

\subsection{Up-sampling}

Up sampling is the process of increasing the sampling rate of a signal. Up sampling images such as photographs means increasing the resolution of the image. Up-sampling enlarges the image, making up additional pixels to fill in the gaps

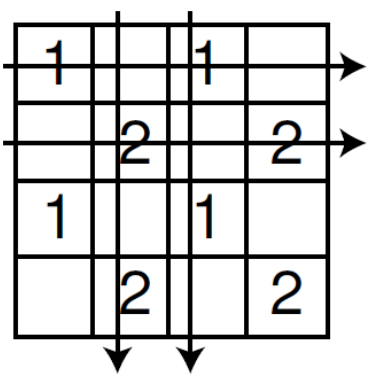

(a)

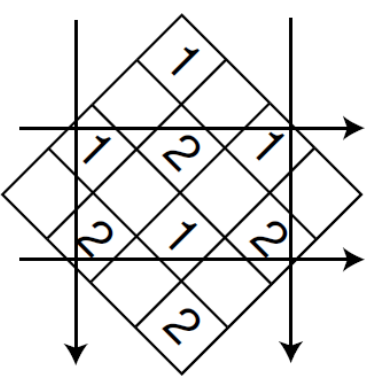

(b)
Figure 3 Rotation (a) high resolution grid (b) $45^{0}$ rotated high resolution grid 
In this method up sampling is done by a factor of 2 . Up sampling creates space for the missing coefficients in the curvelet domain and these coefficients are interpolated. This is done for each rotated sub band high resolution grid. Fig 4 shows the missing locations created during up sampling

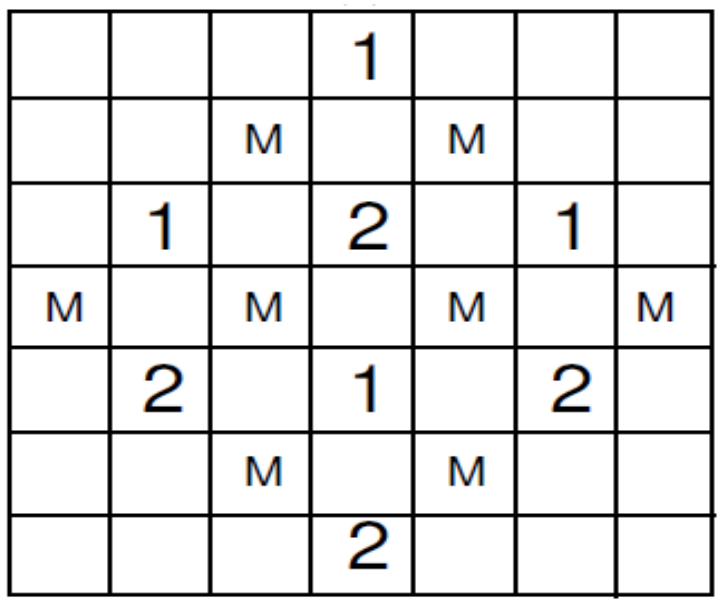

Figure 4 Up sampled rotated high resolution grid

\subsection{Discrete Curvelet Transform}

Curvelet transform[12] is one among directional wavelet transform with anisotropic property (width $\approx$ length $^{2}$ ). Curvelet transform can handle curve discontinuities very well compared to wavelet transform. The needle-shaped elements of this transform possess multiscale with very high directionality and anisotropy. In 2005, Candes proposed two new forms of Curvelet transform based on different operations of Fourier samples, namely, unequally-spaced fast Fourier transform (USFFT) and wrapping based fast Curvelet transform which are simpler, faster, and less redundant than existing technique. Fig. 5 shows the elements of wavelets, curvelets
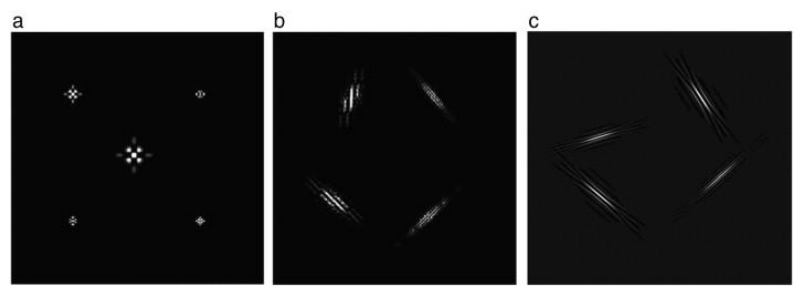

Figure 5 Elements of (a)wavelets (b) curvelets (c) contourlets $[12]$

In this paper we use Curvelet transform based on wrapping. Curvelet transform based on wrapping of Fourier samples takes a 2-D image as input in the form of a Cartesian array $\mathrm{f}[\mathrm{m}, \mathrm{n}]$ such that $0 \leq \mathrm{m}<M, 0 \leq n<N$ and generates a number of Curvelet coefficients indexed by a scale $\mathrm{j}$, an orientation 1 and two spatial location parameters $\left(\mathrm{k}_{1}, \mathrm{k}_{2}\right)$ as output. Discrete Curvelet coefficients can be defined by :

$$
C_{j, l, k_{1}, k_{2}}^{D}=\sum_{\substack{0 \leq m<M \\ 0 \leq n<n}} f[m, n] \varphi_{j, l, k_{1}, k_{2}}^{D}[m, n]
$$

Fig. 6 shows the digital corona of the frequency domain. Basically, wrapping based Curvelet transform is a multiscale transform with a pyramid structure having many orientations at each scale. This pyramid structure consists of several sub bands at different scales in the frequency domain. To achieve higher level of efficiency, curvelet transform is usually implemented in the frequency domain. The process can be described as :

Curvelet transform $=$ IFFT [FFT $($ Curvelet $) \times$ FFT $($ Image $)$.

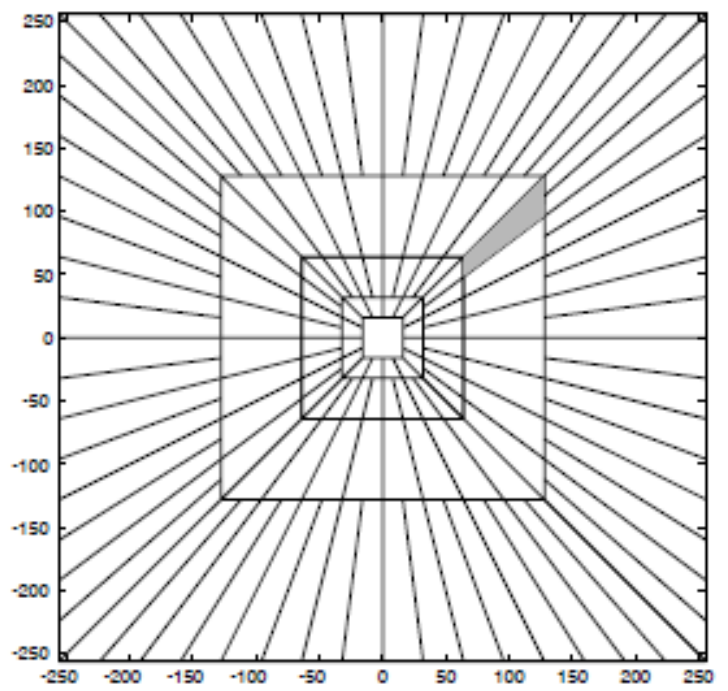

Figure 6 Illustrates the basic digital tiling. The shaded region represents the wedge.

Curvelet transform is performed on each sub bands which is rotated and upsampled. Interpolation is performed on the curvelet coefficients at the finest scale.

\subsection{Interpolation}

Image interpolation refers to the "guess" of intensity values at missing locations. Image interpolation tries to achieve best approximation of a pixel's color and intensity based on the values at the surrounding pixels. Fig. 7 illustrates interpolation

The missing pixel coefficient, $\mathrm{M}$ is calculated using the four nearest neighbors of the missing pixel[8]. The four nearest coefficients of the missing pixel are denoted as $C_{1}, C_{2}, C_{3}$ and $C_{4}$. The missing coefficient is filled using the average of the four nearest neighbors

$$
M=\frac{C_{1}+C_{2}+C_{3}+C_{4}}{4}
$$

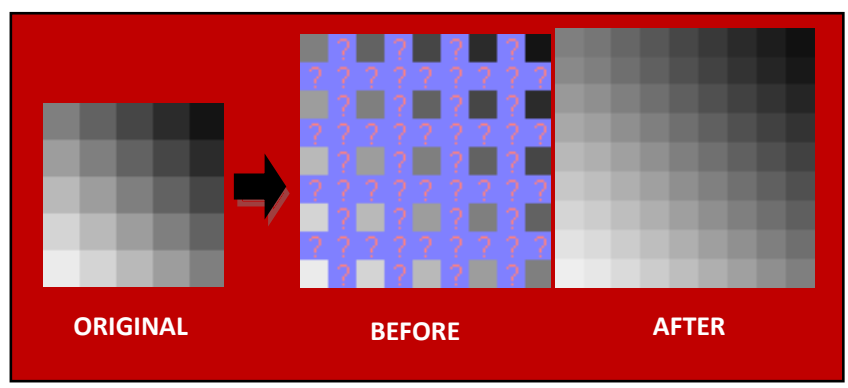

Figure 7 Two dimensional interpolation 
The missing pixel coefficients at the edges are sealed by grouping $C_{1}, C_{2}, C_{3}$ and $C_{4}$. Let $G_{i}$ refer to the groups formed, where i varies from 1 to 4 .

$$
\begin{aligned}
G_{1}=\left\{C_{1}, C_{2}, C_{3}\right\} ; G_{2} & =\left\{C_{2}, C_{3}, C_{4}\right\} ; G_{3}=\left\{C_{3}, C_{4}, C_{1}\right\} \\
G_{4} & =\left\{C_{4}, C_{1}, C_{2}\right\}
\end{aligned}
$$

Calculate the standard deviation of each group and find out the group with minimum standard deviation $\mathrm{K}$ and fill the missing pixel at the edge with the mean of that group. This process is repeated for every missing pixel coefficients in each sub bands of SWT.

$\mathrm{K}=\min \left\{\operatorname{std} \_\operatorname{dev}\left(\mathrm{G}_{\mathrm{i}}\right)\right\}$

$\mathrm{M}=\operatorname{mean}(\mathrm{K})$

Inverse curvelet transform is applied for curvelet coefficients of every sub band. The image is re-rotated 45 degree to obtain the original orientation. Then inverse Stationary Wavelet Transform is applied on these four rerotated interpolated image to get the super resolved image. The pixel values are truncated to the range ( 0 -255).

\section{EXPERIMENTAL RESULTS}

The performance of the proposed algorithm is evaluated using the four images : lena of $512 \times 512$, cameraman of $256 \times 256$, dome of $512 \times 512$ and lifting body of $512 \times 512$. The performance of the algorithm is evaluated using the PSNR value. PSNR is calculated using the equation below

$$
\begin{aligned}
& P S N R=10 \log _{10}\left(\frac{255^{2}}{M S E}\right) \\
& M S E=\frac{I}{M \times N} \sum_{i=1}^{M} \sum_{j=1}^{N}(I(i, j)-\hat{I}(i, j))^{2}
\end{aligned}
$$

where $\mathrm{M}$ and $\mathrm{N}$ are size of image and $I(i, j)$ is the original high resolution image and $\hat{I}(i, j)$ is the super resolved image. The results are shown in table I. Computational complexity of the algorithm increases compared to the existing methods of super resolution..

'Cameraman' image shown in Fig. 8 (a) is reconstructed with the proposed algorithm in Fig. 8(d). The reconstructed image of 'Lena' with the proposed algorithm is shown in Fig. 9(d) .The finer details of the image like curvature of the hat and hair are well reconstructed in the proposed algorithm. Fig. 10(d) is the super resolved image of a 'dome' using the proposed algorithm. Fig.11(d) shows the 'lifting body's 'super resolved image. The performance comparison of the proposed algorithm with SWT and curvelet methods is shown in table I

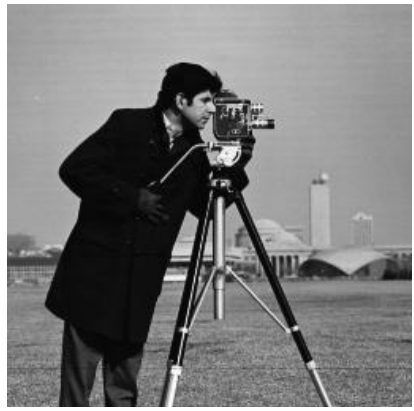

(a)

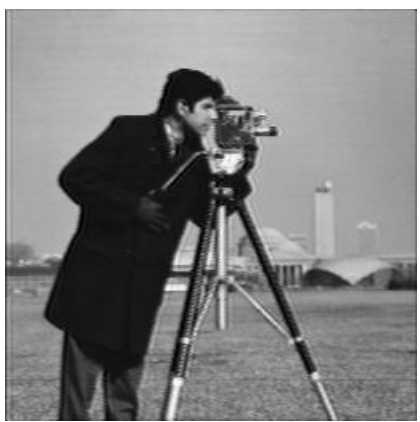

(b)

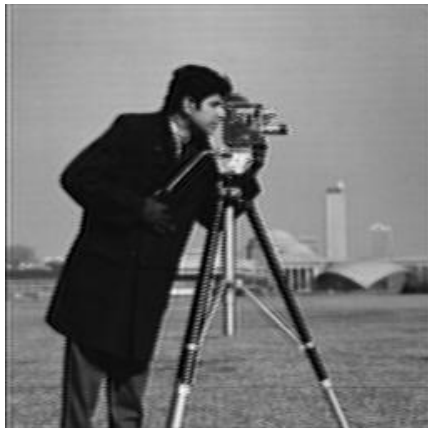

(c)

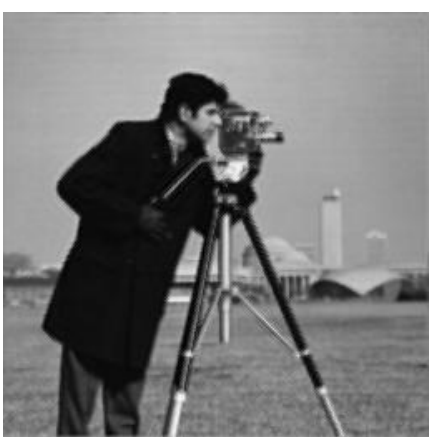

(d)

Figure 8 (a) original image (b) SWT (c) CT (d) proposed method 
TABLE I PERFORMANCE COMPARISON OF ALGORITHM WITH SWT, CuRVElet AND SWT-CuRVELET

\begin{tabular}{|l|l|l|l|l|l|l|}
\hline & \multicolumn{2}{|l|}{ SWT } & \multicolumn{2}{l|}{ CT } & \multicolumn{2}{l|}{ SWT-CT } \\
\cline { 2 - 7 } & PSNR & TIME & PSNR & TIME & PSNR & TIME \\
\hline Lena & 44.5 & 26.43 & 42.7 & 36.76 & 48.11 & 126.6 \\
\hline $\begin{array}{l}\text { Camera } \\
\text { man }\end{array}$ & 40.37 & 5.89 & 43.68 & 7.02 & 48.31 & 25.05 \\
\hline Dome & 35.46 & 28.4 & 37.57 & 38.25 & 38.58 & 132.44 \\
\hline $\begin{array}{l}\text { Lifting } \\
\text { Body }\end{array}$ & 42.95 & 25.29 & 45.93 & 33.84 & 46.49 & 121.13 \\
\hline
\end{tabular}

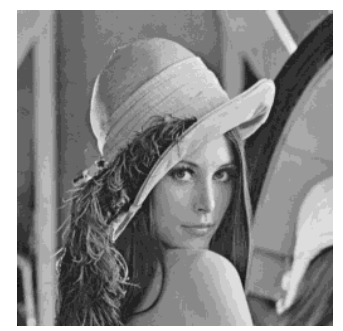

(a)

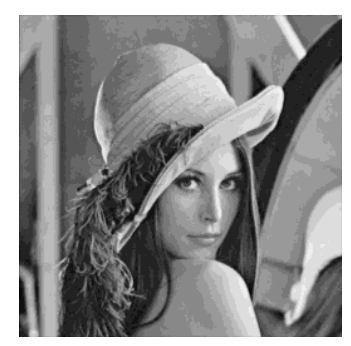

(b)

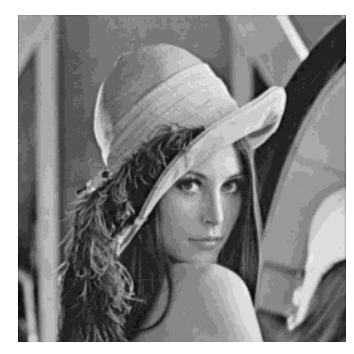

(c)

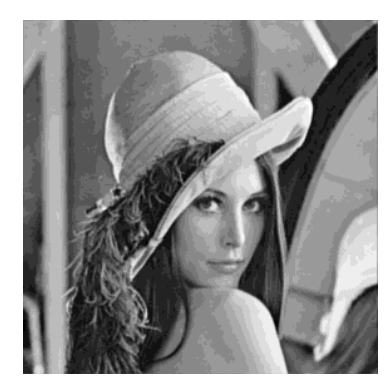

(d)

Figure 9 (a) original image (b) SWT (c)CT(d) proposed method

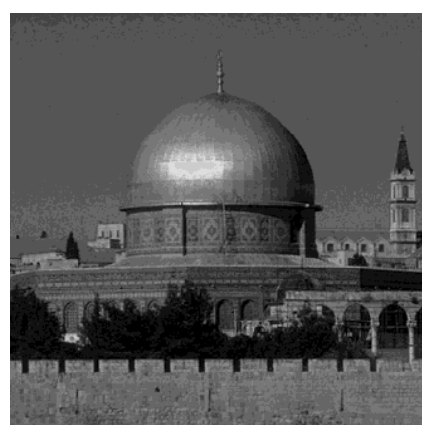

(a)

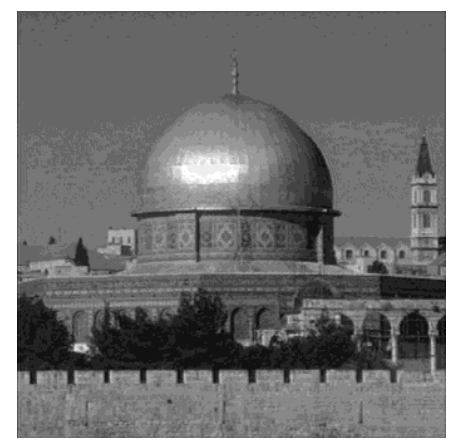

(b)

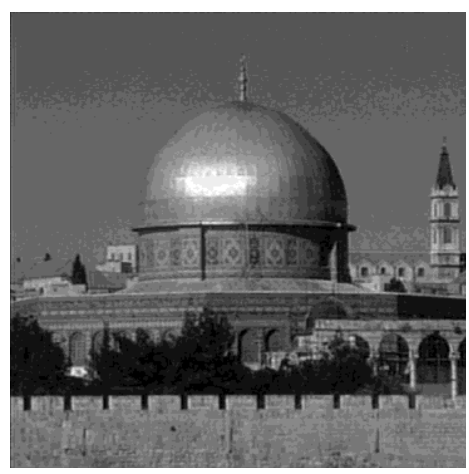

(c)

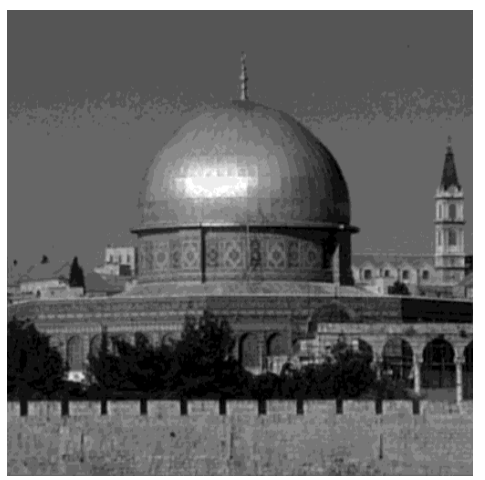

(d)

Figure 10 (a) original image (b) SWT (c) CT (d) proposed method 


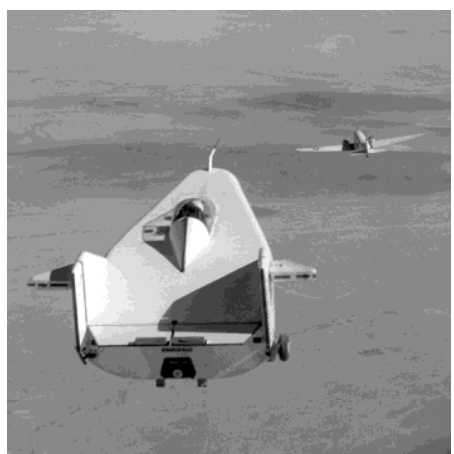

(a)

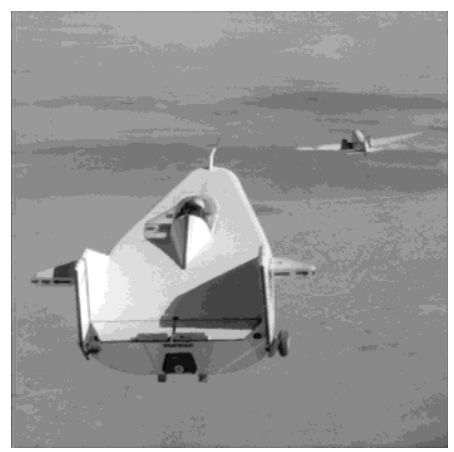

(b)

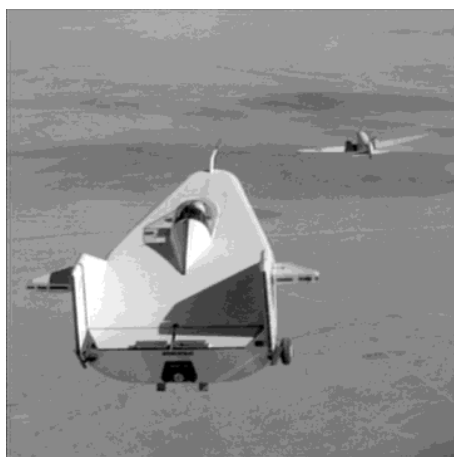

(c)

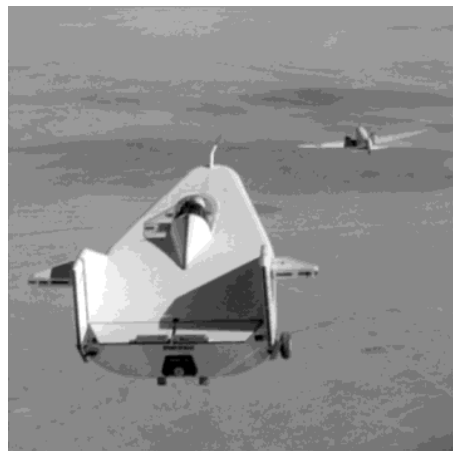

(d)

Figure 11 (a) original image (b) SWT (c) CT (d) proposed method

\section{CONCLUSION}

The proposed algorithm converts two low resolution images into a super resolved image using the features of SWT and CT. HR grid is formed from the low resolution images by SWT and then interpolated in the curvelet domain. This method has the advantages of higher PSNR without using any filter, as compared to the existing methods of super resolution. However, the computational burden has increased. This is because of the use of SWT for the formation of HR grid. In SWT no decimation is performed so there is an increase in computation time. The proposed algorithm needs further research to minimize the time elapsed with good PSNR.

\section{REFERENCES}

[1] Xuelong Li, Yanting $\mathrm{Hu}$, Xinbo Gao, Dacheng Tao, Beijia Ning "A multiframe image super resolution method", signal processing 2010, vol 90, Iss 2, pp 405 414

[2] R Y Tsai, T S Huang," Multiframe image restoration and registration",Advances in computer vision and image processing 1984,vol 1,pp 317-339

[3] K D Sauer, J P Allebach,"Iterative reconstruction of bandlimited images from non-uniformed spaced sample" IEEE Transc Circuits and systems,1987, vol 34,pp 14971505

[4] K Aizawa ,T Komastu,T Saito "Acquisition of very high resolution images using stereo cameras", Proc SPIE visual communication and image processing, Nov 1991,pp 318-328

[5] M.Irani and S Peleg” Improving resolution by Image Registration" CVGIP Graph Models Image Processing,53:324-335,Dec-1993

[6] I Abdou,'Image Restoration of multiple frame sequences" In Proc.SPIE applications of digital image processing X1X,vol2847,pages 192-202,Denver Co,Aug 1996

[7] N.Nguyen , P.Milanfar, "A wavelet-based interpolationrestoration method for Superresolution", Circuits Systems Signal Processing, 2000, vol 19, issue 4, pp. 321-338.

[8] D.L. Ward, "Redundant discrete wavelet transform based super-resolution using sub-pixel image registration", Master's thesis, Air Force Inst. Of Tech Wright-Patterson AFB OH School of Engineering and Management, 2003.

[9] Anil A Patil ,Rakesh Singhai, Jyoti singhai,"Curveelt Transform Based Super resolution using sub pixel image registration",computer science and electronic engineering conference,2010,pp 1-6

[10] Candes E J, Donoho D L and Ying L," Fast Discrete Curvelet Transform", Journal of Multiscale modeling \& Simulation,2006, vol 5, issue 3, pp. 861-899.

[11] G P Nason, B W Silverman, "The stationary wavelet transform and some statistical Applications"Lecture Notes in Statistics, 103, pp. 281-299

[12] Zhang Xiangjun, Wu Xiaolin, "Image coding on Quincunx lattice with adaptive lifting and interpolation", IEEE data compression conference 2007,pp 193-202

[13] Jianwei Mal , Gerlind Plonka, "Computing with Curvelets: From Image Processing to Turbulent Flows",computing in science and engineering, 2009, issue 2, vol 11, pp 72-80 